\title{
Interpolating DFT data for 15D modelling of methane dissociation on an fcc metal
}

\author{
Terry J. Frankcombe \\ School of Physical, Environmental and Mathematical Sciences, \\ University of New South Wales, PO Box 7916, \\ Canberra BC 2610, Australia \\ Research School of Chemistry, Australian National University, \\ ACT 2601 Australia \\ t.frankcombe@adfa.edu.au
}

\begin{abstract}
Detailed simulation of reactions occurring on and with the surfaces of crystalline materials usually require a continuous representation of the potential energy surface that describes the adsorbate-surface interaction. Only a few techniques are available to describe interactions with polyatomic adsorbates that respect all of the symmetries of the interactions. The modified Shepard interpolation has recently been reformulated to ensure symmetries are rigorously imposed. In this work the modified Shepard interpolation is used to construct a $15 \mathrm{D}$ potential energy surface for the reaction of
\end{abstract}


methane with the $\{100\}$ surface of a face-centred cubic metal, in the BornOppenheimer static surface (BOSS) approximation. The energy of the system is calculated using density functional theory and the geometries around which the potential is expanded are selected by quasi-classical trajectory calculations. The energy of the resulting continuous potential energy surface exactly matched the DFT energy at these points; there is no fitting error. It is demonstrated that the classical reaction probability converges with a reasonable number of interpolation points for this $15 \mathrm{D}$ system.

\section{Introduction}

Gas-surface chemistry is vital to the functioning of technological society. ${ }^{1} \mathrm{Re}$ actions occurring on and with surfaces are pervasive in virtually all technological and industrial activities. Being able to predict and thus engineer reactions at surfaces has profound importance, with applications from designing new heterogeneous catalysts for industrial processes to predicting the performance of space vehicles.

Electronic structure theory methods such as periodic density functional theory (DFT) have been quite successful at calculating the energies of adsorbates interacting with metal surfaces. ${ }^{2-8}$ Interactions with non-metallic crystal surfaces are harder to describe with DFT, though recent developments with molecular fragmentation descriptions of adsorbates interacting with wide band gap materials show promise. ${ }^{9,10}$

Irrespective of the success or otherwise of DFT in describing the interactions, calculating interaction energies is only part of the story when considering 
experimentally-realisable behaviour for catalytic systems. The accurate modelling of reactive processes requires the inclusion of the dynamics of the reaction. While it is becoming possible to calculate the required forces "on the fly", ${ }^{11,12}$ for dynamics calculations a continuous representation of the potential energy surface (PES) defined by e.g. DFT is usually required.

As is the case for molecular PESs, there are a number of viable approaches to constructing gas-surface PESs to model catalytic systems. For example, the energies calculated at particular geometries can be fit to an analytic functional form, the PES can be expressed in a neural network or cluster-type representation, and there are several approaches based on local expansions around geometries where the energy and other properties have been calculated explicitly. However, relatively few of these methods have been applied to polyatomic adsorbates (e.g. Refs. 13-23).

One method that uses local expansions is modified Shepard interpolation. Building on the success of modified Shepard interpolation for building the PESs for gas phase reactions in an algorithm often called Grow, ${ }^{24-30}$ modified Shepard interpolation has been used to represent the PES in gas-surface reactions. ${ }^{14,31-34}$ This approach has several advantages, including being able to treat polyatomic adsorbates, simple and seamless treatment of reactive or non-reactive collisions, physisorption, chemisorption and surface catalysed reactions, and strict interpolation of known energies. The modified Shepard interpolation of gas-surface interaction energies has recently been reformulated with a robust adherence to the plane group symmetry of the underlying crystalline surface. ${ }^{35}$

The current work demonstrates the application of the reformulated modified Shepard interpolation to the full-dimensional description of polyatomic adsor- 
bates catalytically reacting on a crystalline surface. For this demonstration we use methane interacting with the $\{100\}$ surface of a face-centred cubic (fcc) metal, such as nickel or platinum. This reaction is a step in the methane steam reforming process that is central to hydrogen production and global agriculture. The catalysed methane dissociation reaction has received a lot of recent theoretical attention ${ }^{11,12,14-16,36-45}$ due to its importance in steam reforming and the intriguing

effects of methane vibrational excitation. ${ }^{46-49}$ With 15 degrees of freedom in the static surface approximation, this presents a challenging test of any PES generation approach.

\section{Methods}

The formalism for constructing a modified Shepard interpolation of a potential energy surface describing the interaction between polyatomic adsorbates and a crystalline solid surface has been described in detail elsewhere. A relatively brief mathematical description shall be given here. Readers are referred to Ref. 35 for further details.

\subsection{Modified Shepard interpolation}

The global gas-surface PES constructed by a modified Shepard interpolation is an interpolation between Taylor series of the PES at a number of "data point" geometries. The potential energy and the first and second derivatives of the potential energy (with respect to the coordinates of the adsorbate atoms) need to be calculated at each of these points. How these points are selected shall be discussed below. 
Three different sets of coordinates are used for various purposes within the modified Shepard interpolation of gas-surface interpolations. The $3 \mathrm{~N}$ Cartesian coordinates of the $N$ adsorbate atoms are denoted $\mathbf{X}$. A set of redundant internal coordinates that allow symmetry operations to be applied as a linear transformation are denoted $\mathbf{Z}$. Around each interpolation data point a set of $3 N$ local internal coordinates are defined, denoted $\zeta(i)$ for the coordinates associated with the $i$ th data point. The geometry of any particular adsorbate configuration (relative to the surface unit cell of the crystalline surface) can be described in any of the $\mathbf{X}, \mathbf{Z}$ or $\boldsymbol{\zeta}(i)$ coordinates. There are well-behaved transformations between each representation. Cartesian coordinates are used for dynamics calculations and for interacting with electronic structure theory (e.g. DFT) programs.

The redundant coordinates $\mathbf{Z}$ comprise three distinct groups. The first are the full set of $N(N-1) / 2$ inverse distances between the $N$ adsorbate atoms. These are the same coordinates used for performing modified Shepard interpolation of the PES of gas phase molecular systems. ${ }^{25,26}$ The second are a set of $N$ inverse heights. These are the inverse of the perpendicular distance between each adsorbate atom and a plane parallel to the surface of the crystal, but set deeper within the crystal than the expected or allowed penetration of adsorbate atoms into the surface layer of the crystal surface. The third set are periodic coordinates describing the projected position of each adsorbate atom onto the surface unit cell. For each atom $i$, we define

$$
\begin{aligned}
& \alpha_{i}=\mathbf{x}_{i}^{T} \hat{\mathbf{a}} \\
& \beta_{i}=\mathbf{x}_{i}^{T} \hat{\mathbf{b}}
\end{aligned}
$$


and, in the case of surfaces with hexagonal symmetry,

$$
\gamma_{i}=\mathbf{x}_{i}^{T} \hat{\mathbf{c}}
$$

where $\hat{\mathbf{a}}, \hat{\mathbf{b}}$ and $\hat{\mathbf{c}}$ are the reciprocal lattice vectors of the crystal surface unit cell and $\mathbf{x}_{i}$ is the vector of Cartesian coordinates of the $i$ th adsorbate atom. The periodic coordinates are then taken as

$$
h_{i}^{2} \cos 2 \pi \chi_{i}
$$

and

$$
h_{i}^{2} \sin 2 \pi \chi_{i}
$$

for $\chi=\alpha, \beta, \gamma$, where $h_{i}$ is the inverse height coordinate for atom $i$. Both sine and cosine terms are required to uniquely define a position within the unit cell. There are $6 \mathrm{~N}$ of these periodic coordinates if the crystal surface is hexagonal, $4 \mathrm{~N}$ otherwise. Together, there are $N_{\text {red }}=N(N+13) / 2$ redundant coordinates $\mathbf{Z}$ for $N$ adsorbate atoms if the surface is of hexagonal symmetry, $N_{\text {red }}=N(N+9) / 2$ otherwise.

To define the local internal coordinates around the geometry of data point $i$ we use the Wilson $B$ matrix. The $\alpha, \beta$ element of the variant of the Wilson matrix used here is given by

$$
B_{\alpha \beta}(i)=\frac{\partial Z_{\alpha}}{\partial X_{\beta}}
$$

for $1 \leq \alpha \leq N_{\text {red }}$ and $1 \leq \beta \leq 3 N$, where the derivative is evaluated at the $\mathbf{X}$ and $\mathbf{Z}$ coordinates of the $i$ th data point geometry. The local internal coordinates are 
formed from the singular value decomposition (SVD) of this matrix,

$$
B(i)=U(i) \Lambda(i) V(i)^{T}
$$

as

$$
\zeta(i)=\Lambda(i)^{-1} U(i)^{T} \mathbf{Z} .
$$

In the vicinity of the geometry of data point $i$, the Taylor series expansion of the PES is expressed as

$$
T_{(i)}(\mathbf{Z})=E(i)+\Delta \mathbf{E}(i)^{T} \Delta \boldsymbol{\zeta}(i)+\frac{1}{2} \Delta \zeta(i)^{T} F(i) \Delta \zeta(i)+\text { higher order terms }
$$

where $E(i)$ is the energy at the data point geometry [equivalently expressed as $\mathbf{X}(i), \mathbf{Z}(i)$ or $\zeta(i)], \Delta \mathbf{E}(i)$ is the vector of first derivatives at data point $i$ with respect to elements of $\zeta(i), F(i)$ is the matrix of second derivatives at data point $i$ with respect to elements of $\boldsymbol{\zeta}(i)$, and $\Delta \boldsymbol{\zeta}_{i}$ is the displacement of the point $\mathbf{Z}$ from the data point geometry $\mathbf{Z}(i)$ in $\zeta(i)$ coordinates. $\Delta \mathbf{E}(i)$ and $F(i)$ are formed by simple transformations of the equivalent Cartesian derivatives. In this work, as in similar gas phase calculations, the Taylor series expansions are truncated at second order. ${ }^{35,50,51}$

The global PES is given by the modified Shepard interpolation of $N_{\text {data }}$ data points, and all symmetry-equivalent copies of these data points,

$$
V(\mathbf{Z})=\sum_{i=1}^{N_{\text {data }}} \sum_{g \in G_{\mathrm{CNP}} \times G_{\mathrm{PG}}} w_{(g \circ i)}(\mathbf{Z}) T_{(g \circ i)}(\mathbf{Z}),
$$

where the $w$ are weight functions and $G_{\mathrm{CNP}}$ and $G_{\mathrm{PG}}$ are the molecular permuta- 
tion and plane group ${ }^{52}$ symmetry groups, respectively, describing the symmetry of the gas-surface interaction. $G_{\mathrm{CNP}}$ is the complete nuclear permutation group for the atoms of the adsorbate (or an appropriate subgroup), while $G_{\mathrm{PG}}$ describes the symmetry of the solid surface the adsorbate is interacting with. The subscript $(g \circ i)$ denotes that the quantity for data point $i$ has been transformed according to the symmetry operation $g$.

The interpolating weight functions $w$ are derived from a primitive weight $v$ by

$$
w_{(i)}(\mathbf{Z})=\frac{v_{(i)}(\mathbf{Z})}{\sum_{j} \sum_{g \in G_{\mathrm{CNP} \times G_{\mathrm{PG}}}} v_{(g \circ j)}(\mathbf{Z})} .
$$

The primitive weight functions are the so-called "one-part" weights

$$
v_{(i)}(\mathbf{Z})=\|\mathbf{Z}-\mathbf{Z}(i)\|^{-2 p}
$$

initially. When a sufficient number of data points are known (of the order of a hundred), we switch to the "two-part" primitive weight function

$$
v_{(i)}(\mathbf{Z})=\left[\varepsilon_{(i)}(\mathbf{Z})^{2 p}+\varepsilon_{(i)}(\mathbf{Z})^{2 q}\right]^{-1},
$$

where $\varepsilon_{(i)}(\mathbf{Z})$ is a local weighted distance in the redundant internal coordinate space,

$$
\varepsilon_{(i)}(\mathbf{Z})^{2}=\sum_{j=1}^{N_{\mathrm{red}}}\left(\frac{Z_{j}-Z_{j}(i)}{d_{j}(i)}\right)^{2},
$$

in which $Z_{j}, Z_{j}(i)$ and $d_{j}(i)$ are the $j$ th elements of $\mathbf{Z}, \mathbf{Z}(i)$ and $\mathbf{d}(i)$, respectively. The vector $\mathbf{d}(i)$ contains the lengths defining a confidence volume for data point $i$, derived from Bayesian arguments. ${ }^{27}$ Here we take $p=16$ and $q=2$ in Eqs. (9) 
and (10).

Unlike many other gas-surface PES representation approaches, the above procedure can be used to describe PESs for collisions of monatomic, diatomic or polyatomic species with crystalline surfaces. Furthermore, reactions proceeding through "direct" heterogeneous catalysis, Langmuir-Hinshelwood, Eley-Rideal or even more complex many-body mechanisms can be readily described. Reactions with a high coverage of an intermediate state cannot yet be described with the correct treatment of the symmetry of the surface. The formalism for describing the plane-group-symmetric PES of an adsorbate interacting with a crystalline surface can be generalised in a straight-forward manner to describe the interaction of an adsorbate interacting with a rigid lattice in $3 \mathrm{D}$, with full space group symmetry. ${ }^{53}$

An important point to note is that the above procedure defines a true interpolation of the input data (energies and derivatives). If the resulting PES function $V$ is evaluated at a geometry of a data point where a DFT calculation has been performed, or at a geometry that is symmetrically equivalent to a data point geometry, then the PES corresponds exactly to the calculated DFT data. This applies not only to the energies, but also to the gradient of the energy with respect to motion of the adsorbate nuclei and the associated force constants (i.e. the second derivatives). Most other common techniques for generating continuous PES functions from discrete input energies feature a fitting error, where the resulting PES function does not reproduce the energies even where they are known. Although DFT energies could be considered in some sense an approximation to the true energy of the system (due to uncertainties in the functional, etc.), the aim of constructing a continuous PES from DFT data must be to reproduce the DFT data where it is known. Avoiding an additional level of approximation (an "approximation to 
an approximation") should be considered a desirable feature in a PES generation method.

\subsection{Grow}

To construct a PES that is satisfactory for performing simulations of gas-surface dynamics, we employ the Grow approach that has been developed by Collins and colleagues. ${ }^{9,10,24-29,31,35}$ After determining the source of the energies to be interpolated (e.g. DFT energies with a particular set of parameters), the first step is to select an initial set of geometries to represent one or more of the expected processes occurring through collisions with the surface. At these geometries the potential energies and the first and second derivatives of the potential energy with respect to all adsorbate coordinates are calculated. This defines an initial interpolated PES through Eq. (7) and the related equations above.

Grow then proceeds iteratively. Classical molecular dynamics simulations of the gas-surface collisions are run on the interpolated PES defined by the current set of data points. From the classical molecular dynamics trajectories, new trajectories are selected in regions where the interpolated PES is suspected of being a poor representation of the underlying potential energies. These trajectory points are selected using the "variance" and " $h$-weight" criteria regularly used in constructing gas phase potentials with the Grow procedure. ${ }^{25}$ The energies and derivatives at these geometries are evaluated and the points added to the set of data points, defining a new and improved interpolated PES. The process is then repeated, beginning with new classical molecular dynamics simulations on the updated interpolated PES. 
An important aspect of Grow is monitoring the convergence of dynamical properties determined from the evolving interpolated PES. This is achieved by periodically performing a more extensive set of molecular dynamics calculations to yield the desired dynamical properties determined with statistical significance. Stabilisation of the examined dynamical properties as data points are added to the interpolated PES is strongly suggestive of convergence of those dynamical properties with respect to the data point sampling. Different dynamical properties can be expected to converge at different points in the Grow procedure. The convergence of dynamical properties should not necessarily be interpreted as being indicative of the convergence of the interpolated PES to the underlying potential.

For both point selection and convergence monitoring, classical trajectories were run with the initial gas phase molecule internal coordinates and momenta selected from a microcanonical distribution using the efficient microcanonical sampling procedure. ${ }^{54}$ The energy was usually selected to mimic the zero point energy (ZPE) of the gas phase molecule, making the calculation a quasi-classical trajectory $(\mathrm{QCT})$ simulation.

Trajectories were integrated until either the incoming molecule had bounced off the surface back into the gas phase region, the incoming molecule dissociated with enough separation between fragments to violate the minimum image convention on the surface supercell (here $2 \sqrt{2} \times 2 \sqrt{2}$ ), or the molecule/molecular fragments remained in contact with the metal surface for a sufficient length of time to deem them to be adsorbed but unreactive. 


\subsection{Density functional theory}

The energy landscape that is being interpolated in this work is defined by density functional theory (DFT). All DFT calculations were performed with the Siesta code. ${ }^{55,56}$ The exchange-correlation functional was the PBE functional and the electron density was allowed to be spin polarised with an electronic smearing parameter of $0.1 \mathrm{eV}$. Siesta's finite-range numerical double zeta plus polarisation pseudo-atomic orbitals were used.

These parameters were selected in part by considering a $\mathrm{CH}_{4}$ molecule in a large box. With the selected parameters and a Hessian matrix determined by finite difference of calculate forces, harmonic vibrational frequencies differ from those calculated at the CCSD(T)/aug-cc-pVQZ level by 3-20 $\mathrm{cm}^{-1}$.

The $\mathrm{CH}_{4}-\mathrm{fcc}(100)$ system is described as a slab under 3D periodic boundary conditions. The fcc lattice parameter was taken to be $3.57 \AA$, the value that minimised the potential energy of the bulk fcc crystal calculated with the same DFT method. The slab was constructed by repeating the crystallographic fcc unit cell, yielding a square surface cell $(7.14 \AA \times 7.14 \AA)$ of eight atoms per layer, with four layers. A $28 \AA$ vacuum layer was inserted between slabs.

The pseudopotential for the fcc metal was nominally that of nickel. However, in this work we do not claim to be modelling a realisable catalysis system, as the energy profile calculated by the Siesta code features a too low barrier to reaction and a spurious deep physisorption well. The key feature of this work is demonstrating the automated construction of a gas-surface PES appropriate for modelling the catalytic decomposition of methane on a metal surface, with all of the interaction energies coming directly from otherwise realistic DFT calculations. 


\section{Results}

All trajectory simulations were initialised with $1.17 \mathrm{eV}\left(43 \mathrm{~m} E_{\mathrm{h}}\right)$ of internal energy in the methane molecule, representing the zero point energy. For each trajectory the initial centre of mass of the methane molecule was set to be $12.2 \AA$ from the plane of the surface atoms of the metal slab. The projection of the methane centre of mass on the slab was selected at random from a uniform distribution. Atoms were considered to be in contact with the metal surface if the normal separation between the atom and the plane of the surface atoms was $2.2 \AA$ or less. Molecules were considered adsorbed if still in contact with the surface $0.16 \mathrm{ps}$ after the $\mathrm{CH}_{4}$ centre of mass velocity normal to the slab had changed sign.

In this work the dynamical quantities being monitored for convergence were the "reaction probabilities" of a $\mathrm{CH}_{4}$-surface collision, being the probabilities that an individual trajectory would result in dissociative adsorption, chemisorption, scattering, etc. For the QCT simulations performed here these quantities are most sensitive to the shape of the "contour" of the PES at the energy that the trajectories are initiated at, being the sum of the initial state equilibrium potential energy, the ZPE and the centre of mass translational energy. This contour defines the classical turning points for the trajectories. Stabilisation of the reaction probabilities as more data points are added to the interpolation suggests that the shape of the PES in general, and the classical turning point contour in particular, are not changing significantly.

The evolution of the calculated probabilities of the observed outcomes of $\mathrm{CH}_{4}-\mathrm{fcc}\{100\}$ collisions is shown in Figure 1. These probabilities are derived by categorising the end state of each trajectory into one of four cases: adsorption 


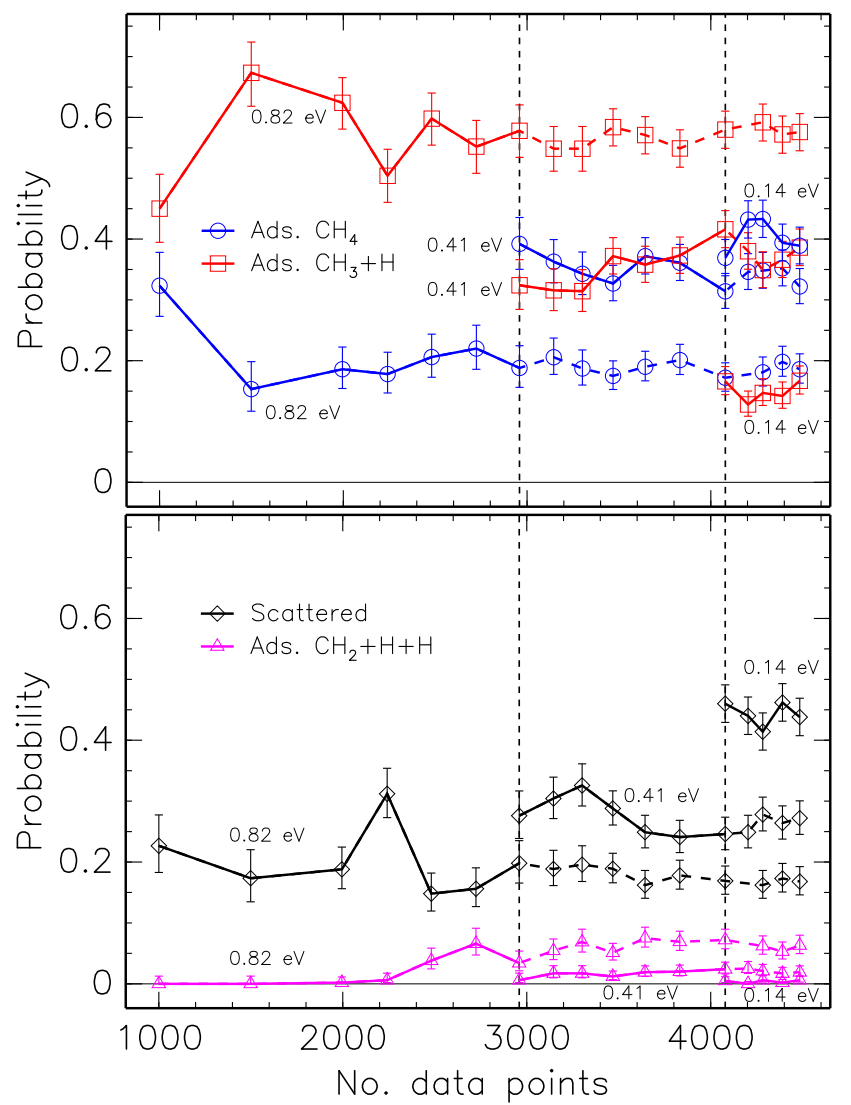

Figure 1: Calculated probabilities for $\mathrm{CH}_{4}-\mathrm{fcc}\{100\}$ collisions as a function of the number of data points in the interpolated PES. Upper panel: $\mathrm{CH}_{4}$ adsorption and dissociative adsorption; lower panel: scattering and double dissociative adsorption. Initial translational energies are indicated. See text for details. 
of the $\mathrm{CH}_{4}$ molecule to the metal surface ("Ads. $\mathrm{CH}_{4}$ "), dissociative adsorption, leaving a $\mathrm{CH}_{3}$ fragment and a $\mathrm{H}$ atom adsorbed on the surface ("Ads. $\mathrm{CH}_{3}+\mathrm{H}$ "), scattering off the surface as $\mathrm{CH}_{4}$ ("Scattered"), or double dissociative adsorption, where two atoms dissociate from the incoming molecule during or shortly after the impact, leaving a $\mathrm{CH}_{2}$ fragment and two $\mathrm{H}$ atoms adsorbed on the surface (“Ads. $\left.\mathrm{CH}_{2}+\mathrm{H}+\mathrm{H} ”\right)$.

The methane centre of mass translational kinetic energy was initially set to $0.816 \mathrm{eV}\left(30 \mathrm{~m} E_{\mathrm{h}}\right)$ for sampling. After the interpolated PES was deemed to be sufficiently converged (approximately 3000 data points), the translational kinetic energy was reduced to $0.408 \mathrm{eV}\left(15 \mathrm{~m} E_{\mathrm{h}}\right)$ and 1100 data points added, before being reduced to $0.136 \mathrm{eV}\left(5 \mathrm{~m} E_{\mathrm{h}}\right)$ to add a further approximately 400 data points. The boundaries between these sampling regimes are shown as vertical dashed lines in Figure 1.

Probabilities for each of the initial translational energies are shown in Figure 1. Batches of trajectories sufficient to determine sticking and reaction probabilities for an initial translational energy of $0.41 \mathrm{eV}$ were only commenced once $0.82 \mathrm{eV}$ sampling was concluded, and similarly for $0.14 \mathrm{eV}$. Thus there are three sets of data evident in Figure 1, one each for initial translational energies of 0.82, 0.41 and $0.14 \mathrm{eV}$, with each starting from a larger number of data points. Batches of trajectories were still run at the higher energies once the sampling energy was reduced. In Figure 1 the probabilities calculated at the energy being used for sampling are connected with solid lines; non-sampled energies are shown with dashed lines.

Using the final 4484 data point interpolated PES, batches of trajectories were run simulating normal incidence $\mathrm{CH}_{4}-\mathrm{fcc}\{100\}$ collisions at initial translational 




Figure 2: Calculated probabilities for $\mathrm{CH}_{4}-\mathrm{fcc}\{100\}$ collisions as a function of initial translational energy.

energies in the range $0.14-0.82 \mathrm{eV}$. The calculated probabilities are shown in Figure 2. While the probability of dissociative chemisorption increases with increasing translational energy, the fractions of molecules being adsorbed as $\mathrm{CH}_{4}$ and bouncing off the surface as $\mathrm{CH}_{4}$ both generally decrease with increasing translational energy.

Figure 3 shows a histogram of the time for $\mathrm{C}-\mathrm{H}$ bond breakage in reactive collisions. The zero of time in this plot is the point at which the $\mathrm{CH}_{4}$ surface normal centre of mass velocity changes sign for the first time, approximately the point of closest approach. The $\mathrm{C}-\mathrm{H}$ bond is taken to be broken when the $\mathrm{C}-\mathrm{H}$ bond length exceeds $1.85 \AA$. The distribution is shown for translational energies of 0.14 and $0.82 \mathrm{eV}$. The distribution for other initial energies is similar.

As a probe of the effect of the initial internal energy added to approximate the methane ZPE, a single batch of trajectories was run with no simulated ZPE. That is, the atoms making up the incoming $\mathrm{CH}_{4}$ molecule were initially in the equilibrium relative positions and given a nonzero velocity only in the direction 


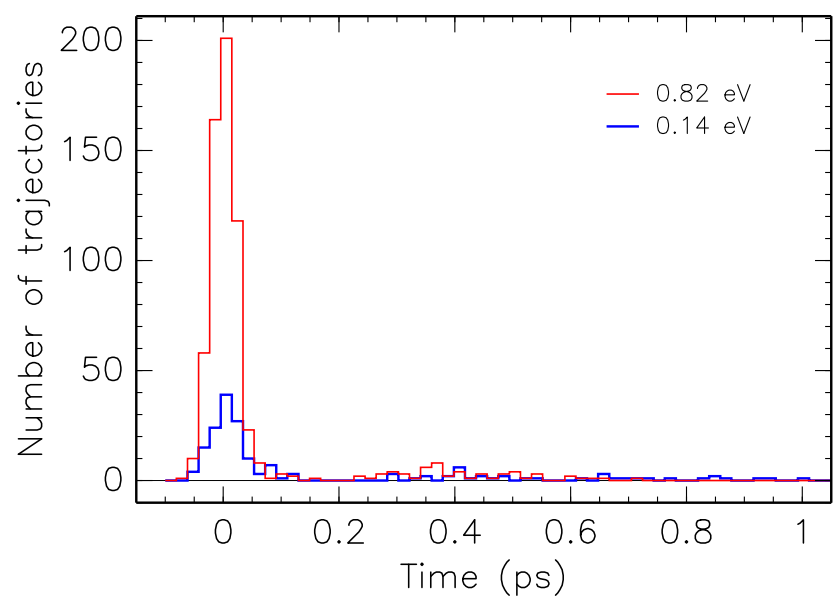

Figure 3: Distribution of delay between the first $\mathrm{CH}_{4}$ centre of mass turning point and $\mathrm{C}-\mathrm{H}$ dissociation.

normal to the metal surface. The reaction probability was greatly reduced from the values shown in Figure 2, with only approximately $3 \%$ of trajectories resulting in $\mathrm{C}-\mathrm{H}$ dissociation. The scattering probability was increased to around $34 \%$.

\section{Discussion}

Using Grow to produce an interpolated PES typically involves sampling from trajectories at some particular total energy (although thermal sampling has been demonstrated, ${ }^{28}$ as well as sampling from quantum wave packets ${ }^{57,58}$ ). It is usually assumed that if dynamical properties such as reaction probabilities are converged at some particular sampling energy, then the PES should be appropriate for describing reactions at a lower energy. That assumption has been tested explicitly in this work, at least for the unconstrained QCT sampling approach used here, by sampling at lower energies after the PES is deemed converged at a particular energy. The results in Figure 1 generally support the view that convergence at 
a particular sampling energy yields appropriate reaction probabilities from QCT calculations at lower energies. There are some cases, such as dissociative adsorption for an initial translational energy of $0.41 \mathrm{eV}$, where the calculated probabilities do change sufficiently to give confidence intervals for the reaction probability that do not overlap with those calculated with the smaller set of data points that was deemed converged for higher energies.

The sampling procedure was reasonably efficient, in the sense that only a small fraction of the data points were not used to construct an energy. In a batch of 1000 trajectories sampled with an initial translational energy of $0.82 \mathrm{eV}$, only 120 of the final 4484 data points did not contribute to a PES evaluation somewhere with a weight [Equation (8)] of at least $10^{-4}$. That the majority of these points (almost 100) were added when sampling at translational energies less than $0.82 \mathrm{eV}$ suggests that there is value in additional sampling at lower energies.

The QCT calculations were performed at a total energy well above the barrier to reaction presented by the DFT method. Were the reaction occurring near threshold, the energetics of the description of the PES in the vicinity of the barrier would be very important. However, even the lowest initial translational energy trajectories are run at a total energy that is $1.3 \mathrm{eV}$ above the asymptotic energy. With this much energy available within the classical dynamics the reaction probabilities are not strongly affected by the details of the barrier in the vicinity of the saddle point. Indeed, during construction of the interpolated PES it was noted that the value of the interpolated PES in the vicinity of the saddle point could change by $0.1 \mathrm{eV}$, without substantial change in the reaction probabilities. As mentioned above, the dynamics was most dependent on the nature of the PES at the energies of the trajectories. Note that even though no sampling was performed that specif- 
ically targeted the barrier region, the final interpolated PES matches the DFT data reasonably in the barrier region, with errors substantially less than $10 \%$ of the barrier height.

There is no a priori robust way to categorise trajectories as resulting in adsorbed $\mathrm{CH}_{4}$ in these simulations. Once "adsorbed" on the surface the $\mathrm{CH}_{4}$ molecule may desorb or dissociate after a delay, thus shifting into one of the other categories of trajectory. This is particularly the case in these simulations with no surface atom motion. Sticking to the surface requires translation to rovibrational $(T \rightarrow V, R)$ energy redistribution. After a $\mathrm{CH}_{4}$ molecule sticks to the metal surface without dissociating a hydrogen atom, internal vibrational redistribution of the available energy can facilitate dissociation at a later time. This is evident in Figure 3. While the majority of dissociation events were "direct", occurring on the initial collision with the surface, an appreciable fraction of the trajectories went on to dissociate hydrogen at times longer than the approximately 0.16 ps that was taken to indicate $\mathrm{CH}_{4}$ molecular sticking. These trajectories were multiple encounter trajectories, where initially the $\mathrm{CH}_{4}$ bounced off the metal surface with enough $T \rightarrow V, R$ energy transfer to trap the $\mathrm{CH}_{4}$ molecule near the surface (but not "in contact" with it). The $\mathrm{CH}_{4}$ molecule would then return to the surface, and subsequently react. Even trajectories in which $\mathrm{CH}_{4}$ was deemed to have stuck to the surface can only be considered dynamically hindered; as there is no energy sink in the simulated system the long time stable state must be either dissociation on the surface or escape to the gas phase. These considerations mean that the data shown in Figure 2 for adsorbed $\mathrm{CH}_{4}$ is indicative at best. In particular, the variation of the probabilities with translational energy can be strongly influenced by the details of criteria used for sticking, maximum trajectory lengths, etc. 


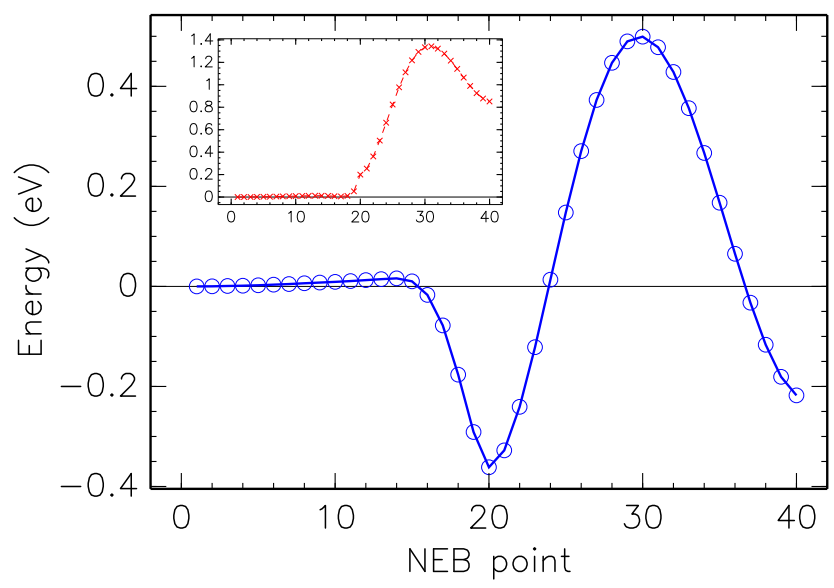

Figure 4: Minimum energy pathway for the $\mathrm{CH}_{4} \longrightarrow \mathrm{CH}_{3}+\mathrm{H}$ reaction on an fcc $\{100\}$ surface, as calculated by Siesta. The reaction proceeds from gas phase $\mathrm{CH}_{4}$ on the left, through a deep adsorption well in the middle to adsorbed $\mathrm{CH}_{3}+\mathrm{H}$ on the right. The inset shows the energies along the same path calculated using the PAW method.

The low initial translational energy reaction probabilities shown in Figure 2 are very large for methane dissociation on a transition metal surface. As mentioned in the Methods section, this is the result of a too-low barrier to reaction calculated using the Siesta code. The nature of the minimum energy pathway (MEP) is revealed in Figure 4. This shows the energies calculated in this work with two nudged elastic band (NEB) calculations, one from gas phase $\mathrm{CH}_{4}$ to the adsorbed $\mathrm{CH}_{4}$ state, and another from the adsorbed $\mathrm{CH}_{4}$ state over the $\mathrm{C}-\mathrm{H}$ dissociation barrier to yield $\mathrm{CH}_{3}$ and $\mathrm{H}$ adsorbed on the surface. Using parameters to give similar quality energies, the potential along the Siesta MEP was calculated with the PAW method ${ }^{59}$ using Vasp. ${ }^{60}$

The PAW energies along the Siesta MEP suggest a barrier to reaction around $1.3 \mathrm{eV}$, much closer to what experimental reactivities suggest than the $0.5 \mathrm{eV}$ predicted by Siesta. That the reverse reaction barriers $(0.5 \mathrm{eV}$ for PAW, $0.7 \mathrm{eV}$ for Siesta) and the differences between the adsorption well geometry and highest 
energies (1.1 eV for PAW, $0.9 \mathrm{eV}$ for Siesta) are quite a bit closer shows that the energy profiles agree more closely for the final part of the reaction path, which might in turn suggest that the Siesta energies are contaminated by a systematic difference between cells in which a slab and a gas phase species are separated and cells in which they are in close contact. Basis set superposition error from Siesta's numerical atom-centred basis functions might be a possible source of such systematic difference, although the magnitude of the difference is large for such an error.

\section{Conclusion}

In this work it has been demonstrated that the recent implementation of modified Shepard interpolation of potential energy data describing adsorbate-surface interactions is practically applicable to polyatomic adsorbates. To the author's knowledge, this is the first method available that provides a true interpolation of energies calculated for polyatomic species interacting with catalytic crystalline surfaces rigorously obeying the plane group symmetry of the crystalline surface. The symmetries of the adsorbate (or adsorbates) arising from the indistinguishability of identical particles (i.e. point group symmetries) are included.

The underlying DFT energies are not sufficiently accurate to consider this work as modelling a particular catalytic surface. Methods such as the specific reaction parameter approach have recently shown promise, but inclusion of, for example, exact/range-separated exchange or dispersion corrections that have recently proved successful in molecular DFT calculations would provide a more predictive theoretical approach. Surface atom motion-which is known ${ }^{61}$ to di- 
rectly affect calculated gas-surface reaction barriers in unpredictable ways-is not included, though it should be noted that work to incorporate surface atom motion into gas-surface interpolations is ongoing. ${ }^{62}$

Instead, this work serves to demonstrate that symmetry-obeying interpolations of energies for gas-surface interactions calculated by electronic structure theory (i.e. periodic DFT) is practical, stable and convergent, even for polyatomic gas phase molecules undergoing catalytic reactions on collision with a catalyst surface.

\section{Acknowledgements}

This work was supported by the NCI National Facility at the ANU. The author acknowledges the support of the Australian Research Council through a Future Fellowship (FT100100824) and Discovery Project grant (DP160100059).

\section{References}

[1] Somorjai, G. A.; Li, Y. Impact of surface chemistry. Proc. Nat. Acad. Sci. 2011, 108, 917-924.

[2] Díaz, C.; Pijper, E.; Olsen, R. A.; Busnengo, H. F.; Auerbach, D. J.; Kroes, G. J. Chemically Accurate Simulation of a Prototypical Surface Reaction: $\mathrm{H}_{2}$ Dissociation on $\mathrm{Cu}(111)$. Science 2009, 326, 832-834.

[3] Díaz, C.; Vincent, J. K.; Krishnamohan, G. P.; Olsen, R. A.; Kroes, G. J.; 
Honkala, K.; Norskov, J. K. Multidimensional Effects on Dissociation of $\mathrm{N}_{2}$ on $\mathrm{Ru}(0001)$. Phys. Rev. Lett. 2006, 96, 096102.

[4] Olsen, R. A.; Busnengo, H. F.; Salin, A.; Somers, M. F.; Kroes, G. J.; Baerends, E. J. Constructing accurate potential energy surfaces for a diatomic molecule interacting with a solid surface: $\mathrm{H}_{2}+\operatorname{Pt}(111)$ and $\mathrm{H}_{2}+\mathrm{Cu}(100)$. J. Chem. Phys. 2002, 116, 3841-3855.

[5] Dong, W.; Hafner, J. $\mathrm{H}_{2}$ dissociative adsorption on $\mathrm{Pd}(111)$. Phys. Rev. $B$ 1997, 56, 15396-15403.

[6] Ferrin, P.; Kandoi, S.; Nilekar, A. U.; Mavrikakis, M. Hydrogen adsorption, absorption and diffusion on and in transition metal surfaces: A DFT study. Surf. Sci. 2012, 606, 679-689.

[7] Groß, A. Ab Initio Molecular Dynamics Simulations of the Adsorption of $\mathrm{H}_{2}$ on Palladium Surfaces. ChemPhysChem 2010, 11, 1374-1381.

[8] Nave, S.; Jackson, B. Methane dissociation on Ni(111) and Pt(111): Energetic and dynamical studies. J. Chem. Phys. 2009, 130, 054701.

[9] Frankcombe, T. J.; Collins, M. A. Potential energy surfaces for gas-surface reactions. Phys. Chem. Chem. Phys. 2011, 13, 8379-8391.

[10] Frankcombe, T. J.; Collins, M. A. Growing Fragmented Potentials for GasSurface Reactions: The Reaction between Hydrogen Atoms and HydrogenTerminated Silicon (111). J. Phys. Chem. C 2012, 116, 7793-7802.

[11] Nattino, F.; Ueta, H.; Chadwick, H.; van Reijzen, M. E.; Beck, R. D.; Jackson, B.; van Hemert, M. C.; Kroes, G.-J. Ab Initio Molecular Dynamics Cal- 
culations versus Quantum-State-Resolved Experiments on $\mathrm{CHD}_{3}+\mathrm{Pt}(111)$ : New Insights into a Prototypical GasSurface Reaction. J. Phys. Chem. Lett. 2014, 5, 1294-1299.

[12] Nattino, F.; Migliorini, D.; Kroes, G.-J.; Dombrowski, E.; High, E. A.; Killelea, D. R.; Utz, A. L. Chemically Accurate Simulation of a Polyatomic Molecule-Metal Surface Reaction. J. Phys. Chem. Lett. 2016, 7, 2402-2406.

[13] Manzhos, S.; Yamashita, K. A model for the dissociative adsorption of $\mathrm{N}_{2} \mathrm{O}$ on $\mathrm{Cu}(100)$ using a continuous potential energy surface. Surf. Sci. 2010, 604, $555-561$.

[14] Krishnamohan, G. P.; Olsen, R. A.; Kroes, G. J.; Gatti, F.; Woittequand, S. Quantum dynamics of dissociative chemisorption of $\mathrm{CH}_{4}$ on $\mathrm{Ni}(111)$ : Influence of the bending vibration. J. Chem. Phys. 2010, 133, 144308.

[15] Jiang, B.; Liu, R.; Li, J.; Xie, D.; Yang, M.; Guo, H. Mode selectivity in methane dissociative chemisorption on $\mathrm{Ni}(111)$. Chem. Sci. 2013, 4, 32493254.

[16] Shen, X.; Chen, J.; Zhang, Z.; Shao, K.; Zhang, D. H. Methane dissociation on $\mathrm{Ni}(111)$ : A fifteen-dimensional potential energy surface using neural network method. J. Chem. Phys. 2015, 143, 144701.

[17] Shen, X.; Zhang, Z.; Zhang, D. H. $\mathrm{CH}_{4}$ dissociation on $\mathrm{Ni}(111)$ : a quantum dynamics study of lattice thermal motion. Phys. Chem. Chem. Phys. 2015, 17, 25499-25504.

[18] Jiang, B.; Guo, H. Dynamics of Water Dissociative Chemisorption on 
Ni(111): Effects of Impact Sites and Incident Angles. Phys. Rev. Lett. 2015, $114,166101$.

[19] Shen, X. J.; Lozano, A.; Dong, W.; Busnengo, H. F.; Yan, X. H. Towards Bond Selective Chemistry from First Principles: Methane on Metal Surfaces. Phys. Rev. Lett. 2014, 112, 046101.

[20] Wonchoba, S. E.; Truhlar, D. G. Embedded Diatomics-in-Molecules Potential Energy Function for Methyl Radical and Methane on Nickel Surfaces. J. Phys. Chem. B 1998, 102, 6842-6860.

[21] Lozano, A.; Shen, X. J.; Moiraghi, R.; Dong, W.; Busnengo, H. F. Cutting a chemical bond with demon's scissors: Mode- and bond-selective reactivity of methane on metal surfaces. Surf. Sci. 2015, 640, 25-35.

[22] Liu, T.; Zhang, Z.; Fu, B.; Yang, X.; Zhang, D. H. A seven-dimensional quantum dynamics study of the dissociative chemisorption of $\mathrm{H}_{2} \mathrm{O}$ on $\mathrm{Cu}(111)$ : effects of azimuthal angles and azimuthal angle-averaging. Chem. Sci. 2016, 7, 1840-1845.

[23] Jiang, B.; Guo, H. Permutation invariant polynomial neural network approach to fitting potential energy surfaces. III. Molecule-surface interactions. J. Chem. Phys. 2014, 141, 034109.

[24] Ischtwan, J.; Collins, M. A. Molecular potential energy surfaces by interpolation. J. Chem. Phys. 1994, 100, 8080-8088.

[25] Collins, M. A. Molecular potential-energy surfaces for chemical reaction dynamics. Theor. Chem. Acc. 2002, 108, 313-324. 
[26] Thompson, K. C.; Jordan, M. J. T.; Collins, M. A. Polyatomic molecular potential energy surfaces by interpolation in local internal coordinates. $J$. Chem. Phys. 1998, 108, 8302-8316.

[27] Bettens, R. P. A.; Collins, M. A. Learning to interpolate molecular potential energy surfaces with confidence: A Bayesian approach. J. Chem. Phys. 1999, $111,816-826$.

[28] Le, H.-A.; Frankcombe, T. J.; Collins, M. A. Reaction Dynamics of $\mathrm{H}_{3}^{+}+$ CO on an Interpolated Potential Energy Surface. J. Phys. Chem. A 2010, 114, 10783-10788.

[29] Zhang, D. H.; Collins, M. A.; Lee, S.-Y. First-Principles Theory for the H + $\mathrm{H}_{2} \mathrm{O}, \mathrm{D}_{2} \mathrm{O}$ Reactions. Science 2000, 290, 961-963.

[30] Godsi, O.; Evenhuis, C. R.; Collins, M. A. Interpolation of multidimensional diabatic potential energy matrices. J. Chem. Phys. 2006, 125, 104105.

[31] Crespos, C.; Collins, M. A.; Pijper, E.; Kroes, G. J. Multi-dimensional potential energy surface determination by modified Shepard interpolation for a molecule-surface reaction: $\mathrm{H}_{2}+\mathrm{Pt}(111)$. Chem. Phys. Lett. 2003, 376, $566-575$.

[32] Crespos, C.; Collins, M. A.; Pijper, E.; Kroes, G. J. Application of the modified Shepard interpolation method to the determination of the potential energy surface for a molecule-surface reaction: $\mathrm{H}_{2}+\mathrm{Pt}(111)$. J. Chem. Phys. 2004, 120, 2392-2404.

[33] Chen, J.-C.; Juanes-Marcos, J. C.; Woittequand, S.; Somers, M. F.; Díaz, C.; 
Olsen, R. A.; Kroes, G. J. Six-dimensional quasiclassical and quantum dynamics of $\mathrm{H} 2$ dissociation on the $\mathrm{c}(2 \times 2)-\mathrm{Ti} / \mathrm{Al}(100)$ surface. J. Chem. Phys. 2011, 134, 114708.

[34] Díaz, C.; Olsen, R. A.; Busnengo, H. F.; Kroes, G. J. Dynamics on SixDimensional Potential Energy Surfaces for $\mathrm{H}_{2} / \mathrm{Cu}(111)$ : Corrugation $\mathrm{Re}-$ ducing Procedure versus Modified Shepard Interpolation Method and PW91 versus RPBE. J. Phys. Chem. C 2010, 114, 11192-11201.

[35] Frankcombe, T. J.; Collins, M. A.; Zhang, D. H. Modified Shepard interpolation of gas-surface potential energy surfaces with strict plane group symmetry and translational periodicity. J. Chem. Phys. 2012, 137, 144701.

[36] Nave, S.; Jackson, B. Methane Dissociation on Ni(111): The Role of Lattice Reconstruction. Phys. Rev. Lett. 2007, 98, 173003.

[37] Tiwari, A. K.; Nave, S.; Jackson, B. Methane Dissociation on Ni(111): A New Understanding of the Lattice Effect. Phys. Rev. Lett. 2009, 103, 253201.

[38] Nave, S.; Tiwari, A. K.; Jackson, B. Methane dissociation and adsorption on Ni(111), $\operatorname{Pt}(111), \mathrm{Ni}(100), \operatorname{Pt}(100)$, and $\operatorname{Pt}(110)-(1 \times 2)$ : Energetic study. $J$. Chem. Phys. 2010, 132, 054705.

[39] Jackson, B.; Nave, S. The dissociative chemisorption of methane on $\mathrm{Ni}(100)$ : Reaction path description of mode-selective chemistry. J. Chem. Phys. 2011, $135,114701$.

[40] Jackson, B.; Nattino, F.; Kroes, G.-J. Dissociative chemisorption of methane 
on metal surfaces: Tests of dynamical assumptions using quantum models and ab initio molecular dynamics. J. Chem. Phys. 2014, 141, 054102.

[41] Mastromatteo, M.; Jackson, B. The dissociative chemisorption of methane on $\mathrm{Ni}(100)$ and $\mathrm{Ni}(111)$ : Classical and quantum studies based on the reaction path Hamiltonian. J. Chem. Phys. 2013, 139, 194701.

[42] Nave, S.; Tiwari, A. K.; Jackson, B. Dissociative Chemisorption of Methane on Ni and Pt Surfaces: Mode-Specific Chemistry and the Effects of Lattice Motion. J. Phys. Chem. A 2014, 118, 9615-9631.

[43] Wang, W.; Zhao, Y. Reaction Rate Constants of $\mathrm{CH}_{4}$ (ads) $\rightleftharpoons \mathrm{CH}_{3}$ (ads) + H(ads) on Ni(111): The Effect of Lattice Motion. J. Phys. Chem. A 2015, $119,12953-12961$.

[44] Campbell, V. L.; Chen, N.; Guo, H.; Jackson, B.; Utz, A. L. Substrate Vibrations as Promoters of Chemical Reactivity on Metal Surfaces. J. Phys. Chem. A 2015, 119, 12434-12441.

[45] Guo, H.; Farjamnia, A.; Jackson, B. Effects of Lattice Motion on Dissociative Chemisorption: Toward a Rigorous Comparison of Theory with Molecular Beam Experiments. J. Phys. Chem. Lett. 2016, 7, 4576-4584.

[46] Juurlink, L. B. F.; McCabe, P. R.; Smith, R. R.; DiCologero, C. L.; Utz, A. L. Eigenstate-Resolved Studies of Gas-Surface Reactivity: $\mathrm{CH}_{4}\left(v_{3}\right)$ Dissociation on Ni(100). Phys. Rev. Lett. 1999, 83, 868-871.

[47] Juurlink, L. B. F.; Smith, R. R.; Killelea, D. R.; Utz, A. L. Comparative 
Study of C-H Stretch and Bend Vibrations in Methane Activation on Ni(100) and Ni(111). Phys. Rev. Lett. 2005, 94, 208303.

[48] Maroni, P.; Papageorgopoulos, D. C.; Sacchi, M.; Dang, T. T.; Beck, R. D.; Rizzo, T. R. State-Resolved Gas-Surface Reactivity of Methane in the Symmetric C-H Stretch Vibration on Ni(100). Phys. Rev. Lett. 2005, 94, 246104.

[49] Juurlink, L. B. F.; Killelea, D. R.; Utz, A. L. State-resolved probes of methane dissociation dynamics. Prog. Surf. Sci. 2009, 84, 69-134.

[50] Jordan, M. J. T.; Thompson, K. C.; Collins, M. A. The utility of higher order derivatives in constructing molecular potential energy surfaces by interpolation. J. Chem. Phys. 1995, 103, 9669-9675.

[51] Crittenden, D. L.; Jordan, M. J. T. Interpolated potential energy surfaces: How accurate do the second derivatives have to be? J. Chem. Phys. 2005, $122,044102$.

[52] Hahn, T. International Tables for Crystallography, 5th ed.; John Wiley \& Sons: Hoboken, 2005; Vol. A.

[53] D’Arcy, J. H.; Jordan, M. J. T.; Frankcombe, T. J.; Collins, M. A. H2 Adsorption in a Porous Crystal: Accurate First-Principles Quantum Simulation. J. Phys. Chem. A 2015, 119, 12166-12181.

[54] Schranz, H. W.; Nordholm, S.; Nyman, G. An efficient microlcanonical sampling procedure for molecular systems. J. Chem. Phys. 1991, 94, 1487-1498.

[55] Soler, J. M.; Artacho, E.; Gale, J. D.; García, A.; Junquera, J.; Ordejón, P.; 
Sánchez-Portal, D. The SIESTA method for $a b$ initio order- $N$ materials simulation. J. Phys.: Cond. Matt. 2002, 14, 2745-2779.

[56] Artacho, E.; Anglada, E.; Dieguez, O.; Gale, J. D.; García, A.; Junquera, J.; Martin, R. M.; Ordejón, P.; Pruneda, J. M.; Sánchez-Portal, D.; Soler, J. M. The SIESTA method; developments and applicability. J. Phys.: Cond. Matt. 2008, 20, 064208.

[57] Frankcombe, T. J.; Collins, M. A.; Worth, G. A. Converged quantum dynamics with modified Shepard interpolation and Gaussian wave packets. Chem. Phys. Lett. 2010, 489, 242-247.

[58] Godsi, O.; Collins, M. A.; Peskin, U. Quantum grow-A quantum dynamics sampling approach for growing potential energy surfaces and nonadiabatic couplings. J. Chem. Phys. 2010, 132, 124106.

[59] Blöchl, P. E. Projector augmented-wave method. Phys. Rev. B 1994, 50, 11169-11184.

[60] Kresse, G.; Furthmüller, J. Efficient iterative schemes for ab initio totalenergy calculations using a plane-wave basis set. Phys. Rev. B 1996, 54, 11169-11184.

[61] Bonfanti, M.; Díaz, C.; Somers, M. F.; Kroes, G.-J. Hydrogen dissociation on $\mathrm{Cu}(111)$ : the influence of lattice motion. Part I. Phys. Chem. Chem. Phys. 2011, 13, 4552-4561.

[62] Frankcombe, T. J. Using Hessian update formulae to construct modified 
Shepard interpolated potential energy surfaces: Application to vibrating surface atoms. J. Chem. Phys. 2014, 140, 114108. 